\title{
Recherches en cours sur le théâtre d'Alésia. Bilan préliminaire (2004-2008)
}

François Eschbach, Sébastien Freudiger et François Meylan

\section{CpenEdition}

1 Journals

Édition électronique

URL : http://journals.openedition.org/edl/78

DOI : $10.4000 /$ edl. 78

ISSN : 2296-5084

Éditeur

Université de Lausanne

\section{Édition imprimée}

Date de publication : 15 mai 2011

Pagination : 29-46

ISBN : 978-2-940331-25-3

ISSN : 0014-2026

\section{Référence électronique}

François Eschbach, Sébastien Freudiger et François Meylan, « Recherches en cours sur le théâtre d'Alésia. Bilan préliminaire (2004-2008) », Études de lettres [En ligne], 1-2 | 2011, mis en ligne le 15 mai 2014, consulté le 18 décembre 2020. URL : http://journals.openedition.org/edl/78 ; DOI : https:// doi.org/10.4000/edl.78 


\section{RECHERCHES EN COURS SUR LE THÉÂTRE D'ALÉSIA BILAN PRÉLIMINAIRE (2004-2008)}

Plus d'un siècle après sa redécouverte, le théâtre d'Alésia a fait l'objet d'une fouille programmée de 2004 à 2008. Ces nouvelles recherches ont été l'occasion de reprendre l'abondante documentation accumulée lors des campagnes précédentes et de vérifier ou de préciser de nombreux points. C'est ainsi que trois états de fonctionnement ont été définis et que l'étude des occupations antérieures à la construction a permis de proposer des hypothèses relatives à l'existence de lieux de réunion ayant précédé le monument actuel. Une synthèse des éléments connus, complétée des nombreux apports issus des dernières fouilles, sera proposée dans la publication du monument. Le présent article en aborde succinctement les grandes lignes.

\section{Introduction}

L'agglomération antique d'Alésia se développe sur le plateau du Mont Auxois (Côte d'Or), culminant à $407 \mathrm{~m}$. Ce site défensif, naturellement protégé par une falaise haute de $20 \mathrm{~m}$, domine la plaine de plus de $150 \mathrm{~m}$. Le théâtre antique se situe au cœur de l'agglomération, à quelques dizaines de mètres du forum, et jouxte le périmètre d'un sanctuaire comportant un temple, peut-être voué à Taranis.

Dégagé à partir de 1905 et fouillé à plusieurs reprises au cours du $\mathrm{XX}^{\mathrm{e}}$ siècle, cet imposant édifice fait l'objet, depuis 2004, de fouilles programmées (fig. 1). Nous présentons ici les principaux résultats de cette opération, sous la forme d'un bilan préliminaire à une publication en préparation. 


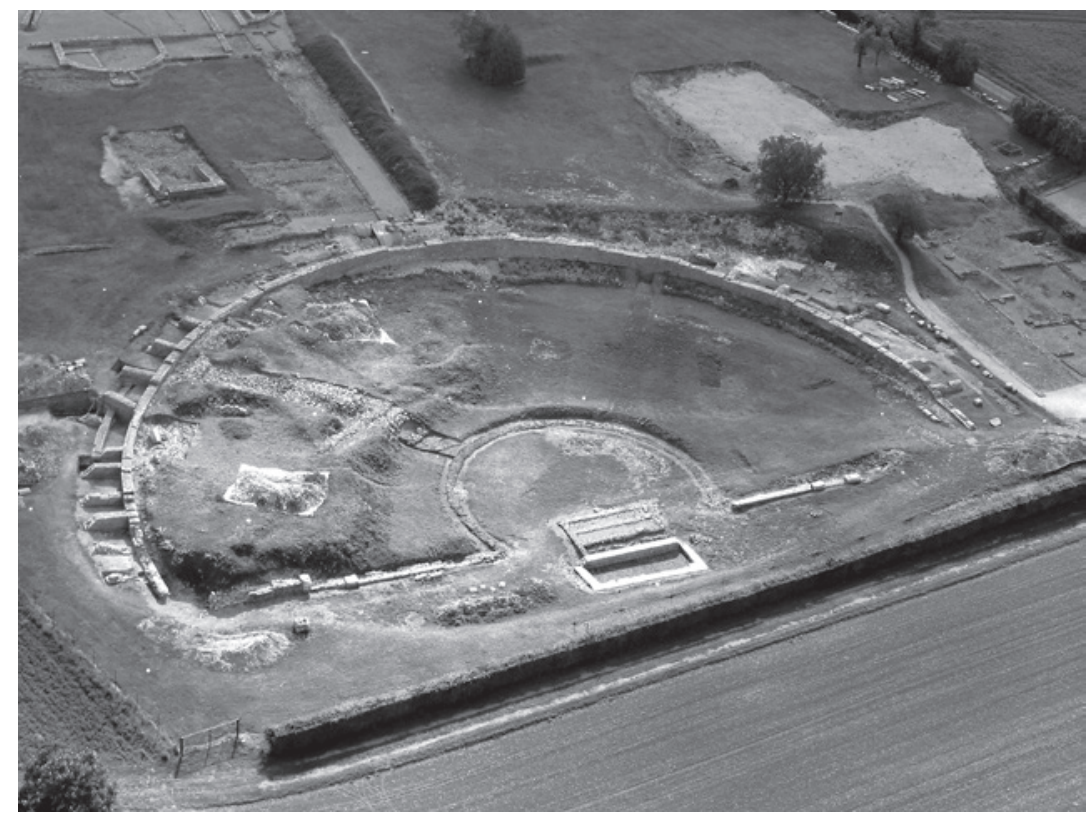

Fig. 1 - Vue aérienne du théâtre en direction du sud. Cliché réalisé pendant la campagne de fouille 2004.

La volonté de reprendre des fouilles sur le théâtre antique remonte à la fin des années 1990 et s'inscrit dans un programme beaucoup plus vaste de mise en valeur globale du site d'Alésia, comprenant la construction d'un nouveau musée et la création d'un parc archéologique. Une opération de fouille programmée sur quatre ans a été mise en place en 2004, sur la base d'un diagnostic archéologique et d'expertises ${ }^{1}$.

Le principal axe de recherche de la publication en cours concerne la définition du plan, la chronologie et l'organisation interne du monument. Les autres thèmes sont l'insertion du théâtre dans le tissu urbain et les occupations antérieures à sa construction.

Après des travaux de débroussaillage et de nettoyage de tous les murs encore debout, une quinzaine de sondages ont été ouverts aussi bien à l'intérieur qu'autour du théâtre.

I. Les campagnes de fouilles ont fait l'objet de rapports annuels, disponibles sur www.archeodunum.ch, et de résumés dans les Bulletins de la Société des Sciences historiques et naturelles de Semur-en-Auxois et des fouilles d'Alésia. 
Les maçonneries du théâtre ont été intégralement documentées par le biais d'une couverture photogrammétrique et par des relevés pierre à pierre des parements, afin de mesurer l'impact des différentes campagnes de restauration du $\mathrm{XX}^{\mathrm{e}}$ siècle et de reconnaître les phases de construction successives. L'analyse de surface a été complétée par des démontages ponctuels et une coupe à travers le mur de la cavea.

De nombreuses perturbations ont affecté le site du théâtre. Que ce soit les importants mouvements de terre occasionnés par les travaux de dégagement du monument au début du $\mathrm{XX}^{\mathrm{e}}$ siècle ou les innombrables fosses d'épierrage, ces phénomènes, le plus souvent mal localisés, ont entraîné la disparition de la plupart des connexions stratigraphiques entre les murs et les couches encore en place. Les principales conséquences sont les difficultés du calage chronologique des structures.

\section{Les occupations antérieures au théatre}

Des traces d'occupations antérieures au théâtre ont été reconnues sur l'ensemble de la zone d'étude (fig. 2). Il s'agit principalement de structures en creux, de murs et murets en pierres sèches et de surface de voirie. Contrairement aux hypothèses de départ, aucune occupation clairement antérieure à l'époque romaine n’a été repérée sur l'ensemble de la zone de fouille ${ }^{2}$.

La probable exploitation des calcaires sous-jacents sur le site du théâtre a été mise en évidence grâce à la découverte de vastes fosses. Elles n'ont jamais été intégralement dégagées; il reste donc difficile d'en exposer le fonctionnement avec précision ${ }^{3}$. La roche, gélive et friable, est exploitable facilement par simple déchaussement, sans recours à un outillage spécifique; cela explique l'absence de toute trace d'outils constatée dans les portions fouillées de ces fosses. Les médiocres qualités de ce matériau ne sont adaptées qu'à la production de moellons de petite taille.

2. Ce constat n'est pas isolé et a été fait lors de campagnes antérieures, notamment lors de la découverte du murus gallicus, à l'extrémité ouest du Mont Auxois. Cf. aussi Ch. Petit, «L'environnement du site d'Alésia», p. 70.

3. Bien que l'hypothèse d'une fonction d'habitat ou de stockage ne puisse être définitivement écartée, elle ne sera pas discutée ici. L'absence de tout indice allant dans ce sens ainsi que les dimensions probables des fosses en sont les principales raisons. 


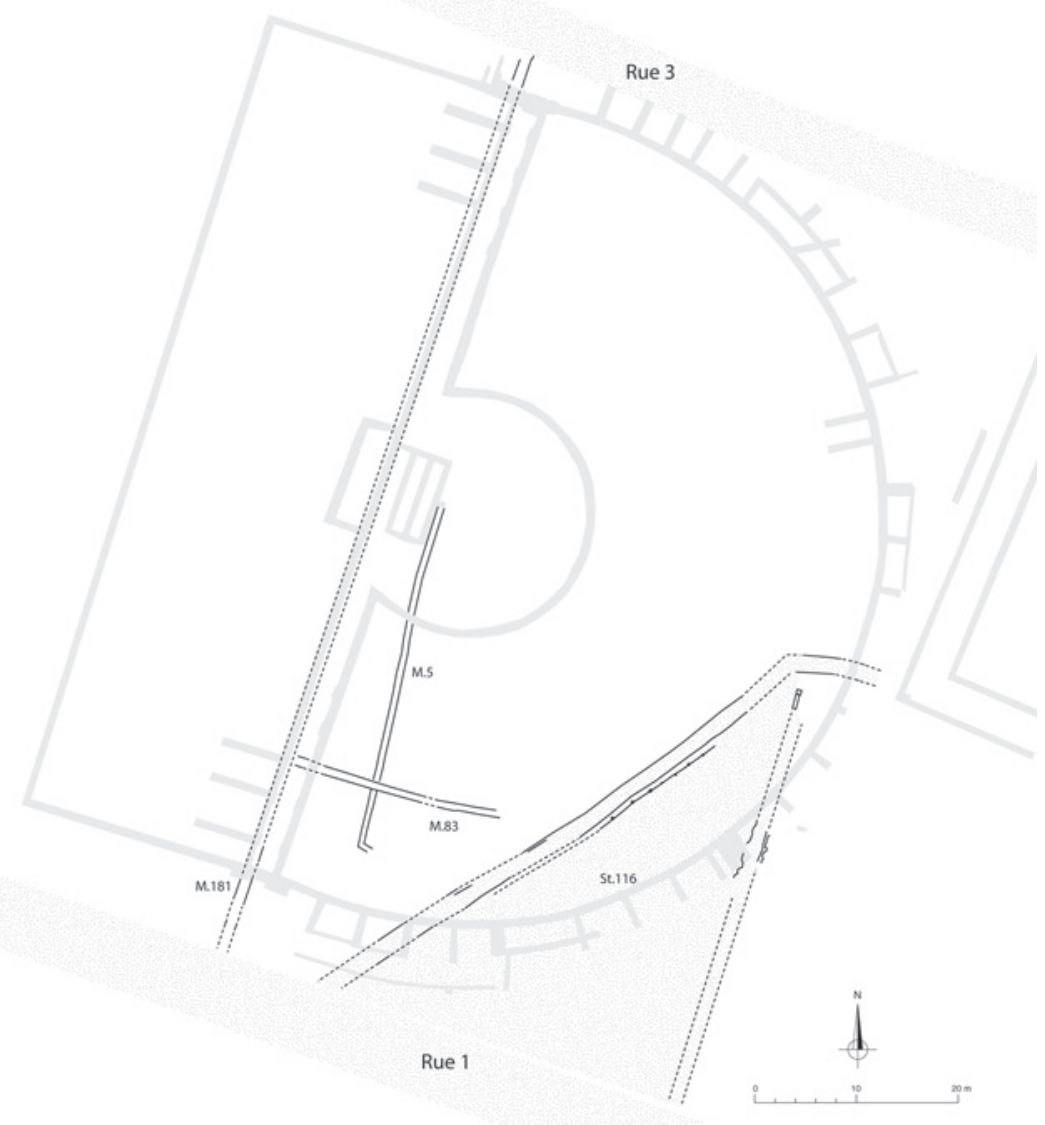

Fig. 2 - Plan des vestiges d'occupation antérieurs au théâtre. 


\subsection{Un assainissement à grande échelle}

De vastes épandages de graviers légèrement émoussés ont été observés systématiquement dans la quasi-totalité des sondages, aussi bien à l'intérieur qu'à l'extérieur du périmètre du théâtre. Les surfaces dégagées n'ont pas permis la détermination définitive de cet aménagement. Il est beaucoup trop ample et accidenté pour pouvoir être considéré comme une «place» d'un seul tenant. Ce «cailloutis» de fond doit être considéré comme un assainissement à grande échelle, peut-être partie intégrante d'un important programme d'urbanisation. En ce sens, il fonctionne en tant que niveau de circulation, même si, curieusement, les clous de chaussures récupérés sur la surface sont rares.

Parmi les structures identifiées, seule une partie des murs de pierres sèches et des fosses mis au jour sous le théâtre pourrait fonctionner en lien direct avec ce niveau.

Le décapage complet du cailloutis sur de grandes zones, sous la cavea, a permis de vérifier qu'aucune structure, hormis les carrières, n'était scellée par cet aménagement. Cette surface empierrée a été fréquentée au moins jusqu'à la fin du règne de Tibère.

\subsection{Des fosses linéaires}

Un grand nombre de fosses percent le cailloutis de fond. Si certaines sont identifiables à des dépotoirs alors que d'autres évoquent des constructions plus élaborées, la plupart demeurent énigmatiques. Néanmoins, quatre ou cinq locaux ont pu être reconnus dans l'espace de la cavea, sans que leur fonction puisse être déduite; peut-être faut-il y voir des lieux de stockage. Compte tenu des surfaces explorées, le bilan est maigre, contrairement à ce qui était attendu et ce d'autant plus quaucune trace d'habitat n'est formellement attestée.

Un certain nombre de trous de poteau fait partie de cette phase de l'histoire du site. Toutefois, avec ou sans calage, la répartition en plan de ces structures n'alimente guère la réflexion.

Le reste des fosses, indépendantes les unes des autres, se distribue selon plusieurs axes privilégiés, sans que l'on puisse en tirer de conclusions définitives. Aucune organisation stricte ne semble avoir dicté l'implantation de ces fosses. On ne constate ni intervalles réguliers, ni regroupements significatifs; de manière générale, aucun soin particulier 
dans la réalisation des creusements n'est relevé. Les nombreux recoupements constatés et le fonctionnement indépendant (?) de chaque structure laissent supposer une utilisation assez brève ainsi que la disparition de leur hypothétique contenu. Le rare mobilier extrait du comblement de quelques fosses ne fournit pas de complément. A ce jour, aucune proposition définitive ne se dégage. Quoi qu'il en soit, ces aménagements condamnent partiellement l'utilisation du cailloutis de fond en tant que niveau de circulation et reposent le problème de sa fonction.

Ces fosses sont creusées et comblées dans une fourchette chronologique comprise entre les règnes de Tibère et de Claude.

\subsection{Les constructions de pierres sèches (M5, M83 et M181)}

Différents types de structures en pierres sèches, distinctement antérieures à l'édification du théâtre, sont apparus à la fouille (fig. 2). Ces constructions ont été reconnues comme des murs de soutènement ou des limites de périmètre. Diversement réparties sur le site, elles ne possèdent pas de lien (attesté) entre elles.

Le plus long de ces murs, M181, a été pointé en plusieurs endroits et pourrait mesurer plus de $80 \mathrm{~m}$.

Reliant les rues d'axe est-ouest bordant la zone d'étude au nord et au sud, il s'agit d'un ouvrage de $110 \mathrm{~cm}$ de largeur, parementé sur ses deux faces, beaucoup plus puissant et de meilleure facture que les autres murs de pierres sèches repérés sous la cavea, avec lesquels il ne possède aucun lien structurel. Ce mur prend appui directement sur le cailloutis de fond et pourrait constituer une clôture. Le fait que le mur de scène du théâtre se superpose ultérieurement à cette structure paraît indiquer le respect d'une limite cadastrale.

Les murs mis au jour sous la cavea sont de piètre facture, ce qui traduit peut-être leur courte espérance de vie propre aux constructions temporaires. L'un d'entre eux, M5, reconnu sur plus de 34 mètres selon un axe nord-sud présente un changement de direction à son extrémité sud. Cette particularité, l'absence d'une détermination définitive et diverses réflexions relatives aux origines du théâtre ont mené à la proposition d'une nouvelle hypothèse d'interprétation pour ces aménagements, la question étant: à quoi succèdent les théâtres? Sur la base des vestiges découverts, la restitution d'un premier lieu de réunion sous les remblais du théâtre à cavea semi-circulaire est une piste qui ne peut plus 
être négligée. Une construction rudimentaire, limitée à une levée de terre contenue derrière des murs de pierres sèches pourrait tout à fait constituer un lieu de spectacle, comparable aux théâtres des origines, en Grèce. Les spectateurs y auraient pris place sur un talus, muni ou non de gradins, face à l'espace réservé aux «acteurs" qui ne comporte pas nécessairement d'aménagement particulier (scène ou décor).

Les remblais retenus par ces murs ont livré du mobilier permettant de proposer une datation entre les règnes de Tibère et de Néron, sans qu'il soit possible d'affiner cette chronologie.

\subsection{Un espace très particulier (St116)}

Déjà repéré lors des sondages de diagnostic, un vaste pavement, aisément identifiable, est apparu directement sous l'humus dans les portions les plus arasées de la cavea (fig. 2). Il s'agit d'une surface constituée de pierres calcaires disposées en hérisson suivant globalement la pente naturelle du terrain d'est en ouest. Afin d'en faciliter la circulation, sa surface est régularisée par un épandage de petits gravillons, repérés ponctuellement.

L'hétérogénéité du sous-sol est à l'origine de certaines particularités de mise en place; ainsi par endroits le pavage cède la place à la roche affleurante.

La limite nord-ouest du pavage, la mieux connue, est bordée d'un cheminement étroit, pavé lui aussi et peut-être couvert d'un portique; c'est sans doute également le cas en périphérie est. Se rejoignant au nord et aboutissant vraisemblablement, au sud, sur un des principaux axes de la cité, les deux cheminements confèrent à la surface pavée un plan triangulaire d'environ $1000 \mathrm{~m}^{2}$. Ce plan singulier au sein de la trame générale de l'agglomération n'est pas encore clairement explicité, mais des alignements dictés par le fonctionnement des terre-pleins situés à l'ouest pourraient en être à l'origine. Cet ensemble se situe dans une fourchette chronologique analogue à celle des constructions en pierres sèches. 


\section{Le théâtre}

\subsection{Un premier projet de théâtre}

Le site retenu pour l'édification du théâtre ne possédait pas la pente nécessaire pour l'aménagement des gradins, si bien que d'importants remblais ont été apportés sur le site. Ces remblais ont livré un abondant mobilier céramique qui permet de les dater de la fin du règne de Claude. Ils sont recoupés par le creusement d'une tranchée curviligne relativement concentrique au mur périmétral du futur théâtre, mais dont le diamètre est légèrement inférieur (fig. 3). Reconnue sur près de la moitié de son tracé supposé, cette tranchée entaille notamment le substrat calcaire et les aménagements liés à la place pavée St116. Le comblement hétérogène de la tranchée est constitué de nombreux blocs calcaires analogues à ceux qui seront utilisés pour le massif de fondation du théâtre. Le mobilier récolté à l'intérieur de la tranchée ne permet pas de proposer une datation différente de celle des remblais de terre. Si l'hypothèse d'un premier théâtre ne peut être définitivement abandonnée, l'absence de traces significatives de construction nous incite à considérer ce creusement comme le tracé abandonné d'un premier projet de théâtre.

\subsection{Premier état}

Les traces du chantier de construction du théâtre ont été mises en évidence sur le pourtour de l'édifice sous la forme de couches de mortier et d'éclats de taille liés à l'édification des murs, et de nombreux trous de poteaux témoignant vraisemblablement de l'installation d'échafaudages le long du mur périmétral. L'examen du mobilier associé aux niveaux de construction permet de le dater du début de la seconde moitié du $\mathrm{I}^{\mathrm{er}}$ siècle de notre ère.

Le théâtre est formé d'une cavea de plan semi-circulaire outrepassé, mais rectifié, qui semble réunir des caractéristiques des monuments de type gallo-romain, grec ou encore romain (fig. 1 et 3 ). Son diamètre de $82 \mathrm{~m}$ permet d'évaluer sa capacité d'accueil à près de 5000 personnes. Amputée des deux tiers de sa surface lors des travaux de fouilles des années 1930, la cavea est composée de remblais de terre supportant un massif de fondation constitué de gros blocs calcaires dont la fonction drainante et les vertus statiques peuvent être soulignées. La 


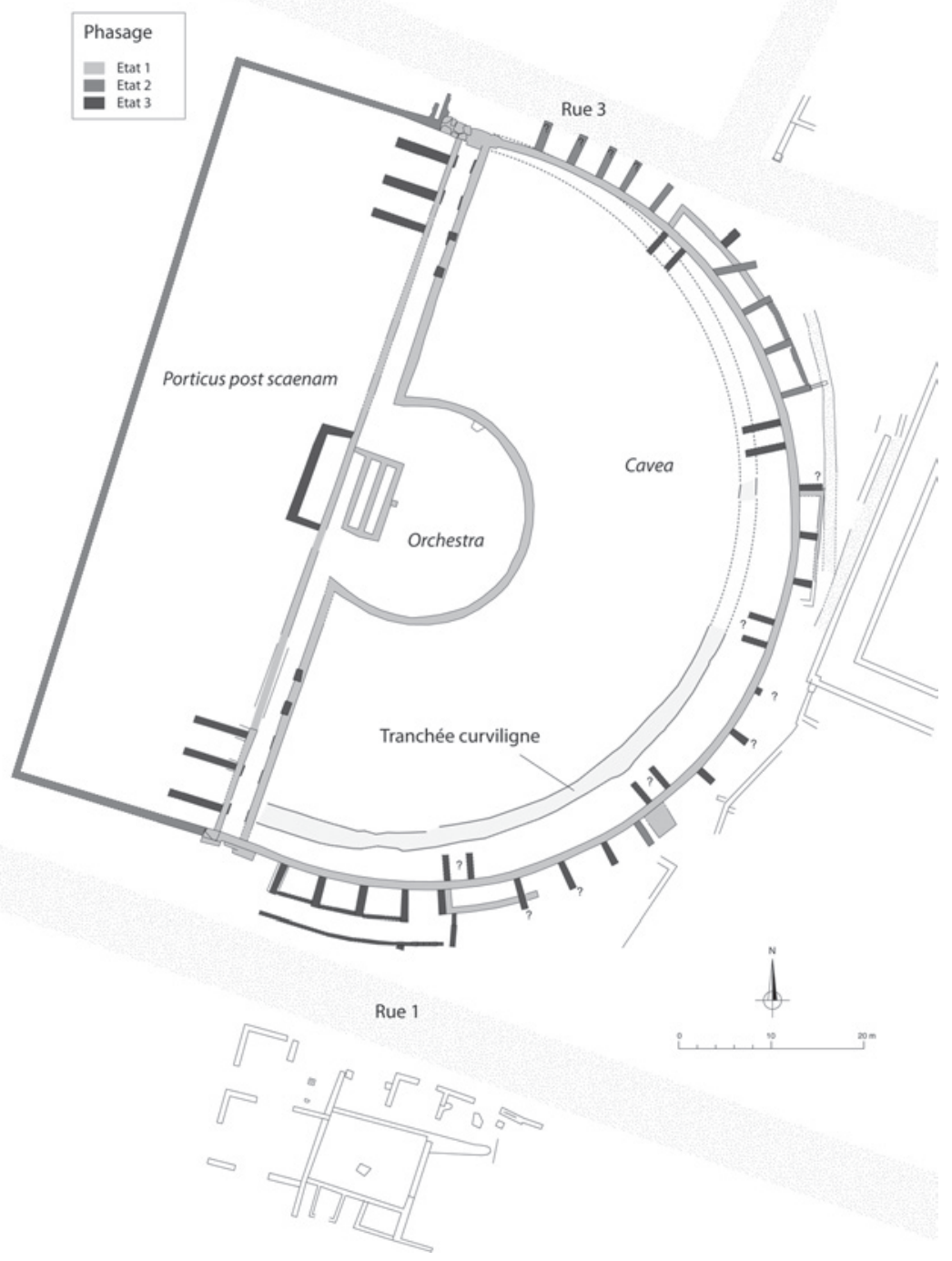

Fig. 3 - Plan du théâtre avec proposition de phasage. 
partie supérieure du massif de fondation a été fortement arasée, si bien qu'aucun vestige de gradins n'a été repéré.

Le mur périmétral qui ceinture la cavea est constitué d'une fondation en hérisson qui repose, selon la morphologie du substrat, sur le rocher, sur d'anciens pavages ou sur des couches argileuses naturelles. L'élévation du mur, épaisse de $90 \mathrm{~cm}$, est constituée d'un petit appareil assez régulier, dont les joints entre les moellons sont tirés au fer et peints en rouge. L'élévation présente un décalage important de son tracé vers l'extérieur par rapport aux fondations, ce qui a produit une dangereuse situation de porte-à-faux au nord de l'édifice en particulier.

L'orchestra, de forme semi-circulaire outrepassée, est délimitée par une fondation fortement arasée. Le dispositif scénique, qui s'avance dans l'orchestra, consiste en une estrade de $9 \mathrm{~m}$ sur 4,20 m, appuyée contre le mur de scène. Elle supportait peut-être un plancher ou une installation plus massive en pierre, comme peut le suggérer la largeur des fondations en hérisson.

Le théâtre est pourvu de deux couloirs latéraux, larges de 2,30 m, longeant les flancs de la cavea sur une distance de $30 \mathrm{~m}$ pour déboucher dans l'orchestra. L'examen de ces couloirs a révélé un état d'arasement important, en particulier lors des transformations de l'état 3, qui a oblitéré les niveaux de circulation. Aux extrémités des aditus, un contrefort en dalles a été aménagé dans le prolongement du mur périmétral curviligne.

Trois dispositifs d'accès adossés à la cavea ont été clairement identifiés. Au nord et au sud sont aménagées deux cages d'escalier montant en direction de l'ouest, et au sud-est, une sorte de rampe dont il ne subsiste plus qu'un massif large de $2,50 \mathrm{~m}$, pour une longueur de près de $3 \mathrm{~m}$. Par symétrie, il est tentant de restituer un quatrième dispositif d'accès au nord-est, mais il n'en subsiste aucune trace. Cette lacune s'explique peutêtre par la disparition d'éléments construits en structures légères.

On ne possède que peu d'informations sur les niveaux de circulation et sur le programme ornemental du monument, en raison de son mauvais état de conservation, des nombreuses restaurations qui ont affecté l'édifice et d'un lapidaire relativement pauvre.

Le théâtre, qui s'intègre dans le tissu orthogonal de l'agglomération, se situe au cœur de la ville antique où se concentrent les édifices civiques et religieux du centre monumental. Au nord, le théâtre est bordé par la rue 3. La vaste surface située au nord-est du théâtre et au sud de la rue 3, semble aménagée par des niveaux de chaussée en cailloutis. Des 
surfaces de circulation sont aménagées à l'est, mais on ne connaît pas précisément leur développement car elles sont recoupées par la construction ultérieure du portique du sanctuaire. Au sud-ouest, la surface de la place pavée semble avoir continué à fonctionner comme chaussée après la construction du théâtre. C'est la rue 1 qui longe le théâtre au sud. L'espace situé entre cette rue et le théâtre ne semble pas avoir été densément aménagé. A l'ouest, les récents résultats de la prospection géophysique renseignent sur l'existence d'une vaste zone qui semble dépourvue de constructions.

\subsection{Deuxième état}

Dans le courant de la première moitié du $\mathrm{II}^{\mathrm{e}}$ siècle de notre ère, des faiblesses dans la structure de l'édifice ont manifestement conduit à ajouter des contreforts contre le parement extérieur du mur périmétral (fig. 3). La nature hétérogène du substrat naturel pourrait constituer une des causes qui a conduit à renforcer l'édifice. Le théâtre se situe en effet à proximité d'une faille géologique qui a provoqué la juxtaposition de banc d'argile au sud-est et de rocher calcaire au nord-ouest. Le dangereux porte-à-faux du mur périmétral a sans doute contribué à fragiliser la cavea où s'exercent les pressions les plus importantes. A ces raisons s'ajoute sans doute aussi l'usage d'un mortier de très mauvaise qualité. Les nouveaux contreforts se concentrent au nord de l'édifice où le substrat argileux est moins propice à la stabilité des murs.

Cette phase de transformation a modifié l'organisation de l'accès nord dont l'extrémité est barrée par un contrefort, alors qu'un tronçon de plus de 4,50 m est amputé par l'implantation d'un second contrefort oblique (fig. 4). Des indices suggèrent cependant que cette cage d'escalier a été réaménagée et maintenue.

La construction des contreforts définit de nouveaux espaces le long du mur périmétral, ce qui entraîne le développement d'une zone d'activité artisanale au nord de l'édifice. Des fosses et des foyers, associés à des battitures et des outils, y attestent une activité métallurgique. En marge de ces transformations est aménagé un trottoir, large de 1,20 m, qui contourne les nouveaux contreforts au nord-est du monument. C'est vraisemblablement à la même époque que sont érigés les portiques qui délimitent le sanctuaire sis à l'est du théâtre. Cet événement provoque sans doute un rétrécissement de la rue orientale. 


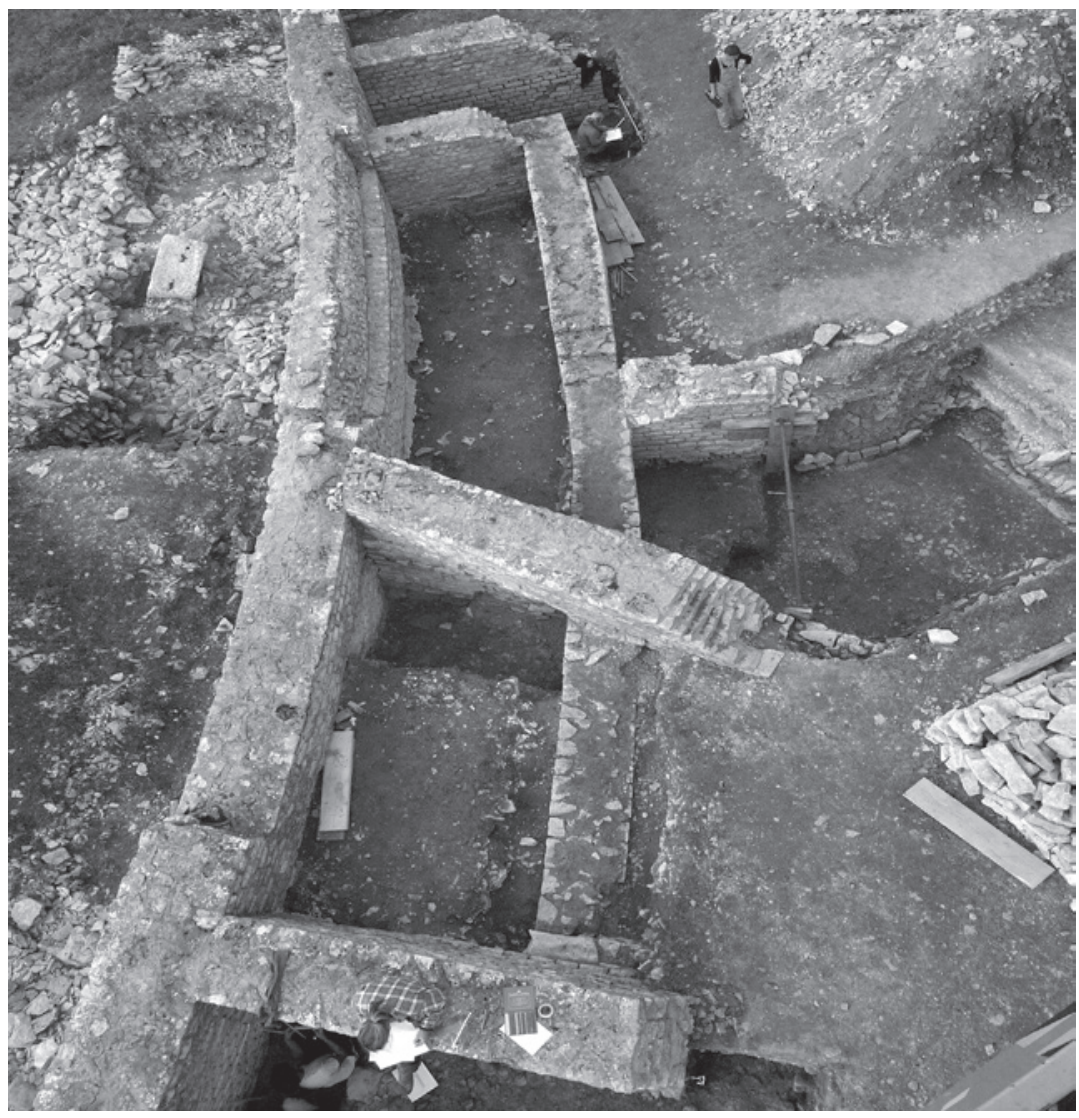

Fig. 4 - Vue générale de la cage d'escalier nord. Au cours du II ${ }^{\mathrm{e}}$ siècle apr. J.-C., des contreforts sont ajoutés contre le mur périmétral. Au nord, leur construction a profondément transformé la cage d'escalier.

Sans que l'on puisse établir de lien direct avec ce programme de renforcement, on perçoit également une volonté de développer le théâtre par l'aménagement d'une porticus post scaenam à l'ouest du monument, dans une période difficile à préciser entre les états 1 et 3 . Ce vaste espace rectangulaire mesure près de $1700 \mathrm{~m}^{2}$. On ignore tout des aménagements internes, mais le mode de construction de ses murs permet de supposer que le niveau de circulation à l'intérieur est inférieur à celui de ses abords. Il pourrait se confondre avec la surface du cailloutis de fond. 


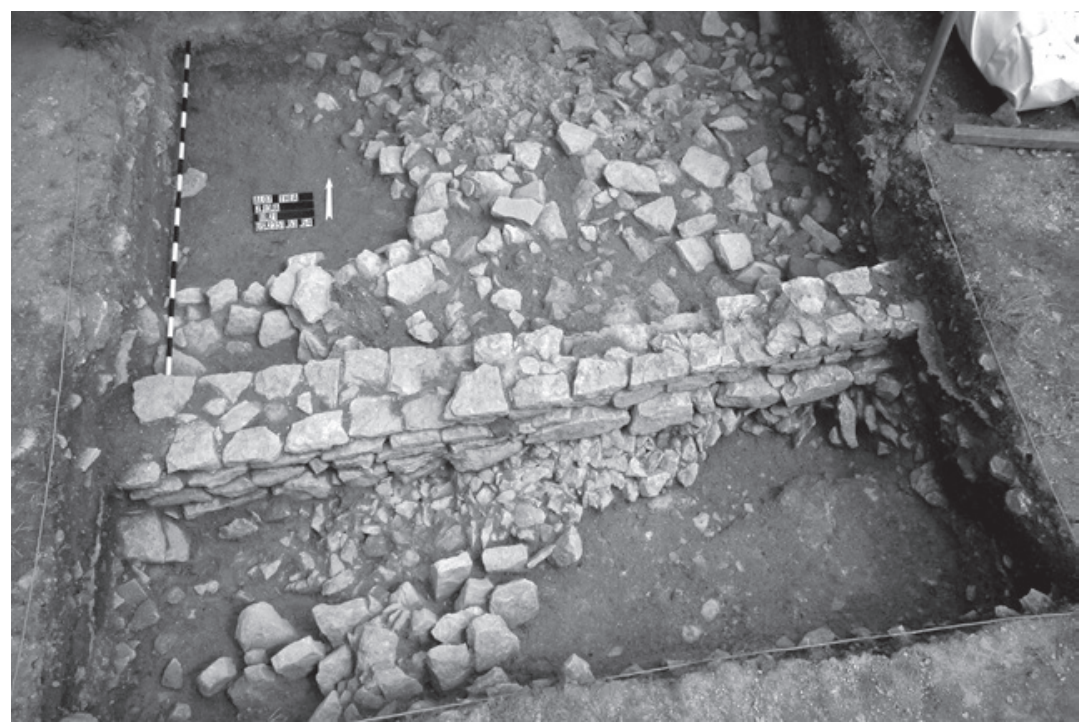

Fig. 5 - Le pan de mur effondré découvert au sud du théâtre correspond selon toute vraisemblance au mur périmétral du premier état de la cavea.

\subsection{Troisième état}

Les transformations du deuxième état se sont toutefois avérées insuffisantes, car le monument est entièrement détruit dans le courant du II $^{\text {e }}$ siècle. Si de nombreuses observations ont confirmé la fragilité des maçonneries, on ne sait pas si cette destruction est accidentelle ou volontaire. Les problèmes évoqués plus haut sont vraisemblablement à l'origine de cet événement. Il est matérialisé notamment par la découverte, au sud de l'édifice, d'un pan de mur effondré dont la nature et la position permettent de l'identifier au mur périmétral (fig. 5).

Le théâtre est donc arasé jusqu'à ses fondations et reconstruit selon un plan identique. L'excellente qualité du mortier utilisé dans les maçonneries du troisième état et la belle facture des parements contrastent avec celles du premier état. Lors de la reconstruction des couloirs latéraux, cinq paires de redans destinés à recevoir un pilier sont aménagées à l'intérieur des aditus (fig. 3). Ces piliers sont associés à la construction de puissants contreforts appuyés contre le mur de scène. Les anciens contreforts du mur périmétral sont reconstruits et de nouveaux murs sont érigés à intervalle régulier sur le reste du pourtour. Le massif de la cavea 
a sans doute été fortement arasé à cette occasion et reconstruit avec de nouveaux couloirs de distribution. Des cinq allées rayonnantes qui peuvent être restituées à cet état, deux ont été observées dans les fouilles anciennes au nord et au sud-est, et une seule est encore conservée au nord-est. Les deux autres sont restituées par symétrie, au droit de la cage d'escalier méridionale, et la plus hypothétique, à l'est, dans le prolongement du portique du sanctuaire. Si les deux cages d'escalier sont conservées à l'état 3 malgré la présence des contreforts, il ne subsiste aucun vestige des dispositifs d'accès attendus en regard des trois autres allées rayonnantes. Il s'agissait peut-être de structures légères qui n'ont pas laissé de traces ou qui ont été arasées. Le mauvais état de conservation des vestiges ne permet pas de mesurer l'impact des reconstructions sur l'orchestra et le dispositif scénique. Des indices suggèrent toutefois que la construction du postscaenium a été entreprise à l'état 3. Après la reconstruction du théâtre se développent des activités artisanales de manufacture du métal entre l'édifice et la rue méridionale, dans l'emprise d'une sorte de portique. Les traces d'une activité de récupération de l'os ont également été détectées au sud-est du théâtre. L'abandon du monument peut être daté approximativement du $\mathrm{IV}^{\mathrm{e}}$ siècle de notre ère.

\section{Synthèse}

Les résultats du programme de recherche en cours sur le théâtre d'Alésia apportent un nouvel éclairage sur la richesse et la complexité de l'histoire de ce secteur de l'agglomération.

Outre l'activité d'extraction du calcaire, c'est principalement l'existence d'un vaste programme d'assainissement, dont la réalisation a peutêtre entraîné la disparition des couches d'époque gauloise, qui domine la perception des premières occupations. Ce programme ne peut malheureusement pas être daté avec certitude, mais les modifications qui vont l'affecter (structures fossoyées, terre-pleins) ne sont pas antérieures au règne de Tibère.

Proposer une interprétation, même approximative, demeure encore impossible pour bien des vestiges postérieurs à cet assainissement initial. Malgré ce flou, on peut affirmer que, préalablement à la construction du théâtre, la zone d'étude n'a pas abrité un quartier d'habitations et/ou d'artisanat analogue à ce qui a été observé au sud et, surtout, à l'est du 
centre monumental ${ }^{4}$. D'autre part, un certain nombre d'éléments structurants peuvent être identifiés. Reliant les principaux axes est-ouest de l'agglomération, le mur nord-sud M181 installe vraisemblablement une division majeure de l'espace, qui sera maintenue par la construction du mur de scène du théâtre. Au sud-est, l'esplanade pavée St116 se distingue par la singularité de son plan, probablement triangulaire, et confère un caractère public, voire monumental, à la zone. L'espace compris entre cette esplanade et le mur M181 a accueilli successivement deux vastes terre-pleins. Si leur fonction demeure énigmatique, le fait qu'un théâtre s'y substitue directement incite à voir dans ces vestiges, sans doute temporaires, les traces de premiers lieux agencés pour des spectacles. La proximité du sanctuaire central, au nord-est de ces vestiges, doit également être prise en considération dans leur perception et leur destination, mais l'exercice se heurte ici à des données lacunaires et disjointes.

Sur le plan chronologique, il importe de souligner le fort dynamisme - qui n'est d'ailleurs pas propre à Alésia - qui caractérise la période située entre la fin du règne de Tibère et le tout début de l'époque flavienne ${ }^{5}$. Dans la zone d'étude, ces quelques décennies voient l'installation de deux terre-pleins successifs, la mise en place de l'esplanade pavée, puis un premier projet de théâtre, et enfin l'édification du théâtre proprement dit.

La compréhension plus détaillée de l'histoire et du fonctionnement du théâtre est un autre apport majeur de ces nouvelles recherches. Les niveaux de travail ont été clairement identifiés et les abondants lots de mobilier, bien stratifiés, ont permis de confirmer la date de construction du monument.

A la différence de ce que l'on pensait jusque-là ${ }^{6}$, les contreforts appuyés contre la cavea se sont avérés appartenir à au moins deux phases de transformations ultérieures. Une première série, au nord du théâtre, semble avoir servi à pallier des faiblesses ponctuelles. Mais la plupart de ces ouvrages de soutènement sont postérieurs à un événement majeur de l'histoire du monument: sa destruction complète et sa reconstruction sur le même plan, dans le courant du $\mathrm{II}^{\mathrm{e}}$ siècle apr. J.-C. Cette datation et les causes et motivations de cette destruction demeurent à préciser. Il est

4. J. Bénard, M. Mangin, "Alise-Sainte-Reine, Alésia», p. 35.

5. Ibid., p. 36-40.

6. A. Olivier, «Le théâtre d'Alésia». 
par ailleurs intéressant de souligner que la construction des contreforts a modifié la configuration de la périphérie de l'édifice et a favorisé l'installation d'activités artisanales dans les loges ainsi créées. On constate donc une imbrication entre la fonction principale du monument, constituant un pôle d'attraction, et des occupations périphériques annexes.

François Eschbach

Sébastien Freudiger

François Meylan

Archéodunum S.A. 


\section{BIBLIOGRAPHIE}

BÉnard, Jacky, Mangin, Michel, "Alise-Sainte-Reine, Alésia», in Les agglomérations antiques de Côte-d'Or, éds Jacky Benard, Michel Mangin, René Goguey, Louis Roussel, Paris, Les Belles Lettres, 1994, p. 28-60.

Eschbach, François, Freudiger, Sébastien, Meylan, François, "Fouilles du Théâtre d'Alésia - Résultats de la campagne 2006", Bulletin de la Société des sciences historiques et naturelles de Semuren-Auxois et des fouilles d'Alésia, 114 (2006), p. 101-107.

—, "Fouilles du Théâtre d'Alésia - Résultats de la campagne 2007", Bulletin de la Société des sciences historiques et naturelles de Semuren-Auxois et des fouilles d'Alésia, 115 (2007), p. 139-147.

Meylan, François, "Un nouveau théâtre à Alésia? Résultats des campagnes 2004 et 2005 sur le théâtre gallo-romain d'Alésia", Bulletin de la Société des sciences historiques et naturelles de Semuren-Auxois et des fouilles d'Alésia, 113 (2005), p. 127-137.

Olivier, Albéric, "Le théâtre d'Alésia: questions posées par la restitution d'un théâtre gallo-romain", in Spectacula II. Le théatre antique et ses spectacles. Actes du colloque tenu au musée archéologique Henri-Prades de Lattes les 27, 28, 29 et 30 avril 1989, éd. Christian Landes, Lattes, 1992, p. 63-70.

Petit, Christophe, "L'environnement du site d'Alésia", in Alésia, éds Michel Reddé, Siegmar Von Schnurbein, Paris, 2001, p. $55-103$.

Crédits iconographiques

Fig. 1-5:

Archéodunum S.A. 
